\title{
Morphology Control of Iron Oxide Nanoparticles
}

\author{
G. Perez ${ }^{*}$, I. G. Solorzano*, M. P. R. Obando**, S. R. W. Louro** \\ *Department of Materials Engineering - PUC, Rio de Janeiro, Brazil - perezgeronimo@hotmail.com \\ ** Department of Physics - PUC, Rio de Janeiro, Brazil
}

Super paramagnetic $\mathrm{Fe}_{3} \mathrm{O}_{4}$ nanoparticles have been studied in several science fields, especially in biomedical applications. Coated magnetic nanoparticles are studied as non-conventional methods in cancer treatment. Magneto hyperthermia is considered as an alternative in cancer therapy [1]. This method claims to selectively destroy cancer cells and to concurrently use the coated magnetic nanoparticles as part of a drug delivery system. The present work aims at establishing the synthesis of the magnetic iron oxide nanoparticles with controlled size and shapes.

The nanoparticles were obtained by co-precipitation from iron salts $-\mathrm{FeSO}_{4}$ and chlorines $-\mathrm{FeCl}_{3}$ in the ratio $\mathrm{Fe}^{2+}: \mathrm{Fe}^{3+}=1: 2$, with the addition of a $\mathrm{NH}_{4} \mathrm{OH}$ solution. There are several factors that control the final size of nanoparticles. In previous research [2], the size of the particles was reported to be $\mathrm{pH}$ controlled. The type of hydroxide used $\left(\mathrm{NaOH}, \mathrm{NH}_{4} \mathrm{OH}\right.$, etc) has an effect on the final size of the particles [3]. Solutions with different concentration of $\mathrm{NH}_{4} \mathrm{OH}(0.15 ; 0.28 ; 0.50$ and 1.M) were added into solutions containing a mixture of $\mathrm{FeCl}_{3}(0.45 \mathrm{M})$ and FeSO4 $(0.225 \mathrm{M})$. The magnetic nanoparticles were washed five times, alternately with water and ethanol in the order to remove residual chemicals reagents; then collected by centrifugation; and finally were magnetically separated. These nanoparticles were washed and dried at $\sim 60^{\circ} \mathrm{C}$ for $12 \mathrm{~h}$ in a furnace. The structure of these nanoparticles was studied by transmission electron microscopy (TEM), scanning electron microscopy (SEM) and X-ray diffraction (XRD). The magnetic behavior was observed by Hall Effect magnetometer. Two different morphologies were observed: faceted nanoparticles and nanowires.

The produced $\mathrm{Fe}_{3} \mathrm{O}_{4}$ nanoparticles were observed by TEM. A Jeol 2010 instrument, operating at $200 \mathrm{kV}$ under diffraction and phase contrast modes was used as main tool. FIG 1 shows typical bright field TEM images of the nanoparticles produced with (a) 0.15 , (b) 0.28 , (c) 0.50 and (d) $1.0 \mathrm{M}$ of $\mathrm{NH}_{4} \mathrm{OH}$, dispersed on a thin carbon supporting film. The sample produced with $0.15 \mathrm{M}$ of $\mathrm{NH}_{4} \mathrm{OH}$ shows elongated shapes around $100 \mathrm{~nm}$ of length (FIG. 1.a), the sample produced with 0.28 $\mathrm{M}$ presents elongated shapes of length around $50 \mathrm{~nm}$ (FIG. 1.b). The samples produced with concentrations of 0.5 and 1.M (FIG.1.c and 1.d), did not present elongated shapes, the particles have an average size in the order of $10 \mathrm{~nm}$ and display a spheroidal morphology. Heterogeneous particle sizes were observed in the aggregate shown in FIG.1.a and FIG.1.b. The population of elongated shapes is seems to be reduced with the increase of $\mathrm{NH}_{4} \mathrm{OH}$ concentrations, and eliminated when concentrations are above $0.5 \mathrm{M}$. In addition, the average size and aspect ratio of the particles is reduced with the increment of the $\mathrm{NH}_{4} \mathrm{OH}$ concentration. Fig 2 shows a typical high resolution image of a $60 \mathrm{~nm}$ long nonowire obtained with an aberration corrected TEM, Zeis Libra at $80 \mathrm{kV}$. This nanowire produced with $0,28 \mathrm{M}$ of $\mathrm{NH}_{4} \mathrm{OH}$ exhibits atomic planes with about $0,37 \mathrm{~nm}$. In summary, TEM has proved to be a fundamental tool in the controlled synthesis of $\mathrm{Fe}_{3} \mathrm{O}_{4}$ nanoparticles via co-precipitation.

References:

[1] R. Hergt, et al., J. Magn. Magn. Mater. 270 (2004) 345.

[2] J. P. Jolivet, et al., C. R. Geoscience 338 (2006) 488-497. 
[3] G. Gnanaprakash, et al., Materials Chemistry and Physics 103 (2007) 168-175.

[4] This research was supported by the National Council for Scientific and Technological Development (CNPq)

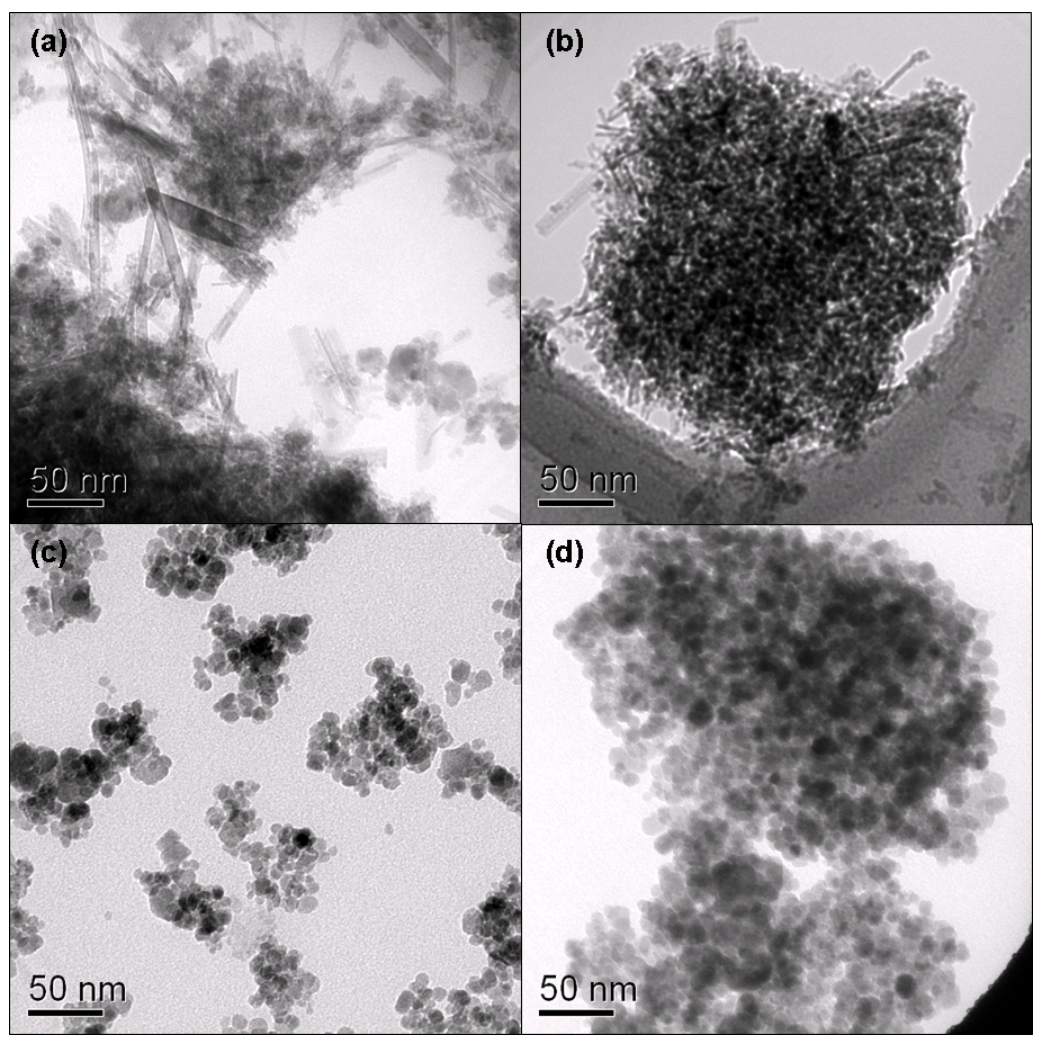

FIG. 1. Bright field micrographs of the nanoparticles produced with (a) 0,15 , (b) 0,28 , (c) 0,50 and (d) $1,0 \mathrm{M}$ of $\mathrm{NH}_{4} \mathrm{OH}$, it shows variation of size and shape of the particles.

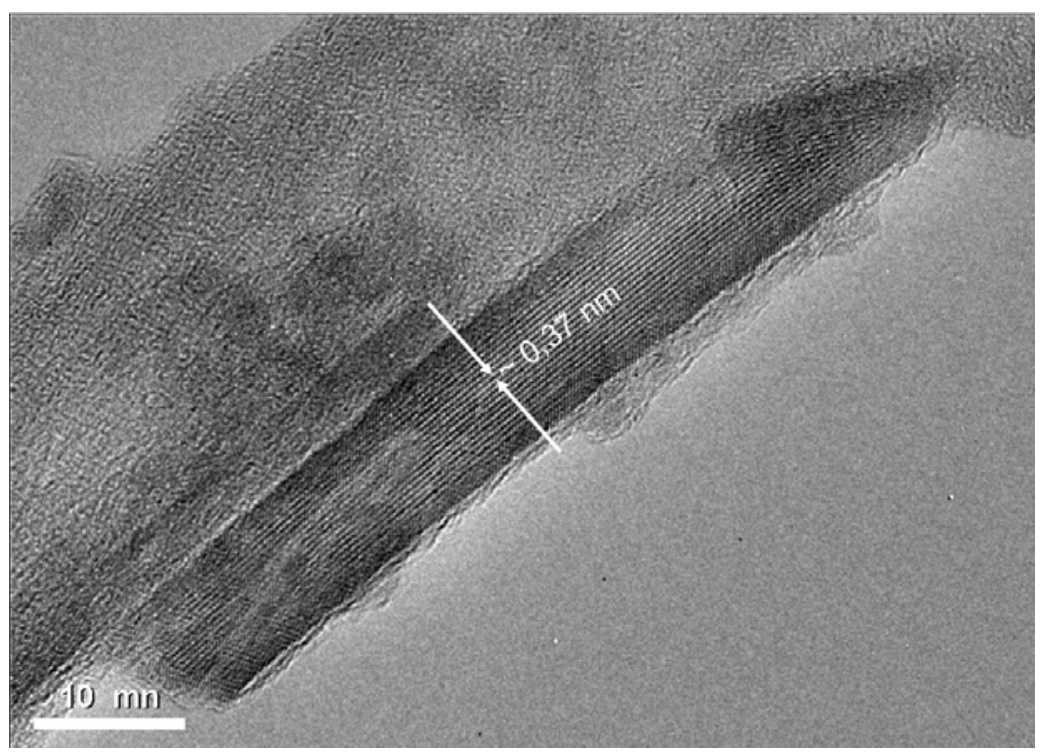

FIG. 2. HRTEM image of $60 \mathrm{~nm}$ nanowire in a sample produced with concentration of $0,28 \mathrm{M}$ of $\mathrm{NH}_{4} \mathrm{OH}$. Atomic planes about $0,37 \mathrm{~nm}$ spacing can be observed. 